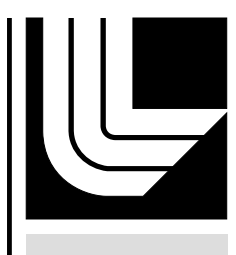

LAW RENCE LIVERMORE N A T IO N A L LABORATORY

Tc with AsqTad and p4rhmc, July 20, 2007 Update

Ron Soltz, Pavlos Vranas, Rajan Gupta

July 24,2007 
This document was prepared as an account of work sponsored by an agency of the United States Government. Neither the United States Government nor the University of California nor any of their employees, makes any warranty, express or implied, or assumes any legal liability or responsibility for the accuracy, completeness, or usefulness of any information, apparatus, product, or process disclosed, or represents that its use would not infringe privately owned rights. Reference herein to any specific commercial product, process, or service by trade name, trademark, manufacturer, or otherwise, does not necessarily constitute or imply its endorsement, recommendation, or favoring by the United States Government or the University of California. The views and opinions of authors expressed herein do not necessarily state or reflect those of the United States Government or the University of California, and shall not be used for advertising or product endorsement purposes.

This work was performed under the auspices of the U.S. Department of Energy by University of California, Lawrence Livermore National Laboratory under Contract W-7405-Eng-48. 


\section{Tc with AsqTad and p4rhmc, July 20, 2007 Update}

\section{Background}

We present the ongoing analysis of Lattice Quantum Chromodymanics runs on the LLNL BG/L supercomputer. This installment adds the density analysis of the p4rhmc for the first few thousand trajectories and the $\psi-$ bar $\psi$ history for hot and cold starts with two values of beta.

\section{P4RHMC Time Histories}

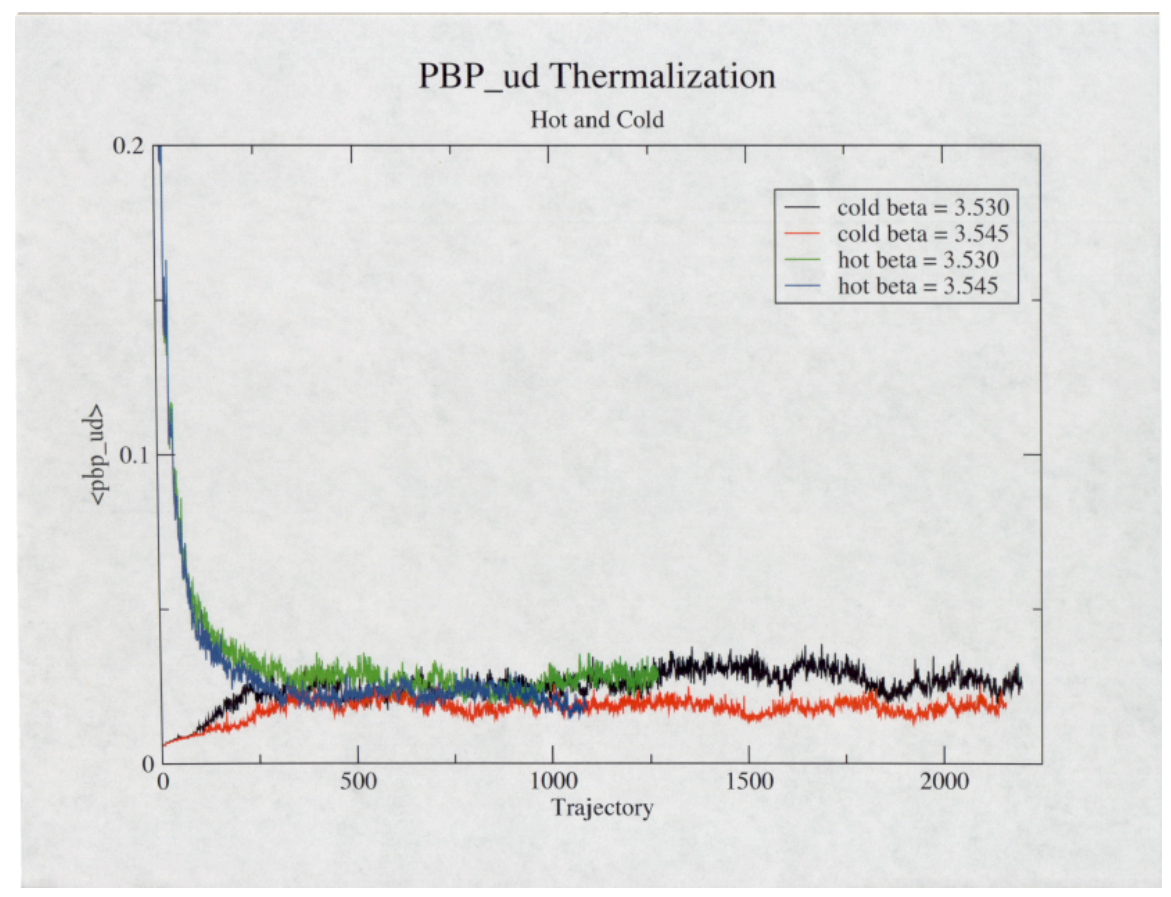

Figure 1: Mean $\psi-$ bar $\psi$ for beta values near $T_{C}$ with two hot and two cold starts

\section{A P4RHMC analyses}

1: $826: \mathrm{p} 4 \mathrm{~b} 3.46100800$

2: $713:$ dens 37500

3: 4946: dens 3.130000e-03 $10.073858689910 .00022321393713 .43719997747752 \mathrm{e}-057.92437172842451 \mathrm{e}$

4: 5743: dens 3.130000e-03 2 -5.56075959345993e-05 3.36817509691821e-05 $7.88578343983395 e-054.59$

5: 5172: dens $3.130000 \mathrm{e}-03 \quad 3 \quad 0.28028708263 .17553100221246 \mathrm{e}-05 \quad 1.90113697514282 \mathrm{e}-052.64847936575$

6: 3941: dens 3.130000e-03 4 -3.563103482 0.017678403510 .016618344820 .01488389511

7: 4270: dens 3.130000e-03 $80.27735134450 .0002854164431-0.00017267216430 .0002724129381$

8: 4842: dens $3.130000 \mathrm{e}-0210.15563189420 .0001582403674 \quad 4.5985389927395 \mathrm{e}-06 \quad 4.47922315885092 \mathrm{e}-0$

9: 5670: dens $3.130000 \mathrm{e}-022-4.67442205012347 \mathrm{e}-06 \quad 1.79638708055408 \mathrm{e}-05 \quad 3.87978733791022 \mathrm{e}-051.573$

10: 5175: dens 3.130000e-02 $30.2787272552 \quad 2.2528011396402 \mathrm{e}-05-1.16029716693944 \mathrm{e}-06 \quad 1.30465822168$

11: 4219: dens 3.130000e-02 $4-2.5267109270 .001670127475-0.00063449056310 .0004681974001$

12: 5148: dens 3.130000e-02 $80.2773431065 .67554427334725 e-05 \quad 5.27636805554923 e-053.649413597098$ 
13: 2339: c_2 3.130000e-03 -0.10772355440 .08835707808$

14: 2978: dens_pbp 3.130000e-03 0.043576627050 .002641254714

15: 2650: chi_dis 3.130000e-03 276.9608892 16.84963199

16: 2663: chi_con 3.130000e-03 2.102231054 0.1276870999

17: 2589: chi_tot 3.130000e-03 279.0631203 16.9758008

18: 2332: c_2 3.130000e-02 0.014565168930 .01504289213

19: 2996: dens_pbp 3.130000e-02 0.091822817570 .005559423575

20: 2589: chi_dis 3.130000e-02 1229.286777 74.4586619

21: 2734: chi_con 3.130000e-02 1.4907594470 .09025088884

22: 2660: chi_tot 3.130000e-02 1230.777537 74.54883578

23: 2924: c11 3.130000e-03 3.130000e-03 -0.95348686910 .1847140985$

24: 2958: c11 3.130000e-03 3.130000e-02 -0.22057548860 .06931661509$

25: 2963: c11 3.130000e-02 3.130000e-02 -0.11771670980 .03079873375$

26: $1645: c 2 q-0.54174768620 .3536686007$

27: $1668:$ c2B -0.17727997620 .05475088895$

28: $1604:$ c2Q 0.17816629150 .01283404338

29: 1625 : c2I 0.73803976030 .04729758986

30: $0:$

31: $829: p 4 b 3.49100800$

32: $705:$ dens 43000

33: 4928: dens 2.900000e-03 $10.053010247850 .00025259138415 .58802966003587 e-056.327749169950916$

34: 5476: dens 2.900000e-03 2 1.97562851670715e-05 3.290508455307e-05 $-4.223459020043 e-063.47204$

35: 5225: dens 2.900000e-03 $30.28022033362 .32210847918671 e-05-1.53047282396535 e-051.6856336478$

36: 4011: dens 2.900000e-03 $4-3.8312614080 .02104081051-0.0010466635410 .01405794665$

37: 4307: dens 2.900000e-03 $80.27420814590 .0002668679488-0.00011505146270 .0001976974126$

38: 4826: dens 2.900000e-02 $10.13170999730 .00017001624672 .99037613573135 \mathrm{e}-05 \quad 3.5279877669022 \mathrm{e}-\mathrm{c}$

39: 5688: dens 2.900000e-02 $28.00445199263443 \mathrm{e}-07 \quad 1.80074584930208 \mathrm{e}-05-4.94710854076918 \mathrm{e}-061.3$

40: 5270: dens 2.900000e-02 $30.27900944981 .78665501543416 e-05-3.73488686967408 e-069.883710362$

41: 4189: dens 2.900000e-02 $4-2.6135259240 .001907240987 \quad 0.00011636176620 .0004487892501$

42: 5243: dens 2.900000e-02 $80.27607620987 .93265662316335 \mathrm{e}-05-3.11969385872501 \mathrm{e}-053.496526136$

43: 2368: c_2 2.900000e-03 0.056977839790 .05744119553

44: 2979: dens_pbp 2.900000e-03 0.032360907120 .001685167434

45: 2636: chi_dis 2.900000e-03 147.7661713 7.790076961

46: 2666: chi_con 2.900000e-03 2.338851441 0.1219606238

47: 2651: chi_tot 2.900000e-03 150.1050228 7.908258978

48: 2376: c_2 2.900000e-02 0.059152120510 .008114991371

49: 2991: dens_pbp 2.900000e-02 0.08040435884 0.004170760499

50: 2604: chi_dis 2.900000e-02 911.016247247 .30034901

51: 2724: chi_con 2.900000e-02 1.5954664070 .08274315128

52: 2645: chi_tot 2.900000e-02 912.611713647 .38293414

53: 2858: c11 2.900000e-03 2.900000e-03 -0.63908026740 .116374652$

54: 2925: c11 2.900000e-03 2.900000e-02 -0.1841901650 .03766697786$

55: 2989: c11 2.900000e-02 2.900000e-02 -0.09163062013 0.01478342315

56: $1734: \mathrm{c} 2 \mathrm{q}-0.0069834182510 .2272429552$

57: $1702:$ c2B -0.092705866370 .03345275668$

58: 1586: c2Q 0.20071022440 .01202857278

59: 1593: c2I 0.75303594690 .04200393403

60: 0 :

61: 822:p4b3.51 100800

62: 712:dens 27500 
63: 4854: dens 2.590000e-03 $10.04010047070 .00031073334726 .41584355218583 \mathrm{e}-058.85809593351321 \mathrm{e}$ 64: 5667: dens 2.590000e-03 $24.38002842518366 \mathrm{e}-05 \quad 4.41533195837226 \mathrm{e}-05 \quad 4.36738976882418 \mathrm{e}-05$ 3.96s 65: 5167: dens 2.590000e-03 $30.28037243942 .88350311957685 \mathrm{e}-052.08805307181416 \mathrm{e}-052.6002054642$ : 66: 3933: dens 2.590000e-03 $4-4.1027429820 .03959038107 \quad 0.017739003110 .01755012149$

67: 4592: dens 2.590000e-03 8 $0.27208385390 .00029888914098 .24338094539328 \mathrm{e}-050.0003483203678$

68: 4881: dens 2.590000e-02 $10.11304014450 .00018910656349 .90365793347717 \mathrm{e}-064.94706622763667 \mathrm{e}-$ 69: 5609: dens 2.590000e-02 2 3.44922695513225e-05 2.40712659401883e-05 2.3508965189699e-05 1.726 70: 5224: dens 2.590000e-02 $30.27936275822 .01576304460025 e-05-1.20204116803679 e-051.509844779$ 71: 4257: dens 2.590000e-02 $4-2.7008408980 .002964006406-0.00028266099930 .0007720949871$

72: 5163: dens 2.590000e-02 $80.27468474198 .69972080529305 \mathrm{e}-05 \quad 5.48603025245131 \mathrm{e}-05 \quad 5.1434487295$ 73: 2249: c_2 2.590000e-03 0.1167670930 .04653309691

74: 2984: dens_pbp 2.590000e-03 0.021326159420 .001866121783

75: 2630: chi_dis 2.590000e-03 73.712561696 .523103656

76: 2661: chi_con 2.590000e-03 2.181913313 0.1913346503

77: 2570: chi_tot 2.590000e-03 75.8944756 .708816345

78: 2352: c_2 2.590000e-02 0.077796615940 .01124418156

79: 2916: dens_pbp 2.590000e-02 0.06011680410 .005240902271

80: 2645: chi_dis 2.590000e-02 584.591286850 .99160563

81: 2683: chi_con 2.590000e-02 1.436356296 0.1252065789

82: 2652: chi_tot 2.590000e-02 586.027643151.11666952

83: 2963: c11 2.590000e-03 2.590000e-03 $-0.4133899113 \quad 0.09407650321$

84: 2987: c11 2.590000e-03 2.590000e-02 $-0.1179633744 \quad 0.03749866493$

85: 3014: c11 2.590000e-02 2.590000e-02 -0.079514845720 .01904221292$

86: $1539:$ c2q 0.18495506040 .182009487

87: 1689: c2B -0.037553984240 .02716189212$

88: 1568: c2Q 0.1784861420 .01648986218

89: $1602:$ c2I 0.64692409740 .05805639001

90: 0 :

91: $825: p 4 b 3.54100800$

92: 705 : dens 20500

93: 4900: dens 2.400000e-03 $10.019465434360 .00037731169452 .02333337548243 e-059.11148135492605$ 94: 5567: dens 2.400000e-03 2 -3.848361585073e-05 4.13269821404141e-05 2.38384206150773e-05 3.670 95: 5225: dens $2.400000 e-0330.28047395793 .22547107011857 e-05-3.82311712982937 e-052.118605399$ 96: 3983: dens 2.400000 e-03 4 -5.008756727 $0.05330438307 \quad 0.0036063821330 .01680421887$

97: 4254: dens 2.400000 e-03 80.26485945250 .00027069550960 .00027524848150 .0002098275603

98: 4902: dens 2.400000e-02 $10.092305857550 .00019989662752 .11003506473275 \mathrm{e}-056.45333072005406$ 99: 5672: dens 2.400000e-02 $2-4.51072411030381 \mathrm{e}-06 \quad 2.81959815613147 \mathrm{e}-051.35602530497238 \mathrm{e}-052.45$ 100: 5125: dens $2.400000 \mathrm{e}-0230.27966856462 .1473375731417 \mathrm{e}-05-3.16126500186145 \mathrm{e}-051.835594193^{\prime}$ 101: 4184: dens 2.400000e-02 $4-2.8187253990 .004955375789-0.00051695620110 .000708201024$ 102: 4873: dens 2.400000e-02 $80.26975600360 .00014992114846 .93667365574226 \mathrm{e}-05 \quad 5.51531860821701$ 103: 2293: c_2 2.400000e-03 $0.2068417418 \quad 0.03698171772$ 104: 3029: dens_pbp 2.400000e-03 0.0089018754710 .001138943231

105: 2634: chi_dis 2.400000e-03 15.03559931 1.988458086

106: 2687: chi_con 2.400000e-03 2.290589967 0.2907465715

107: 2645: chi_tot 2.400000e-03 17.32618927 2.264335753

108: 2267: c_2 2.400000e-02 0.12979500830 .02040063611

109: 2965: dens_pbp 2.400000e-02 0.042213044610 .005340344686

110: 2626: chi_dis 2.400000e-02 335.1988956 42.42413248

111: 2600: chi_con 2.400000e-02 1.28905125 0.1630690142

112: 2653: chi_tot 2.400000e-02 336.487946942 .58704501 
113: 2975: c11 2.400000e-03 2.400000e-03 -0.18932473980 .05796902955$

114: 2890: c11 2.400000e-03 2.400000e-02 $-0.1073093953 \quad 0.0344136572$

115: 2957: c11 2.400000e-02 2.400000e-02 -0.08436868560 .02725364336$

116: 1581 : c2q 0.37035705320 .1150829383

117: 1642: c2B 0.015503884620 .01906385364

118: $1607:$ c2Q 0.18332917690 .02335853034

119: 1591: c2I 0.60300822340 .07673275192

120: 0 :

121: 828:p4b3.57 100800

122: $700:$ dens 20000

123: 4569: dens 2.120000e-03 $10.010423017620 .0002387525297 \quad 0.00010402635496 .51312477382512 e-05$

124: 5705: dens 2.120000e-03 $2-2.52053630006725 \mathrm{e}-05 \quad 3.06225682255356 \mathrm{e}-05-2.01531532865078 \mathrm{e}-052$

125: 5207: dens 2.120000e-03 $30.28048439363 .04979589097566 \mathrm{e}-051.27182618943539 \mathrm{e}-052.184130319$

126: 4078: dens 2.120000e-03 $4-4.2939405020 .047692539870 .0095256796810 .007198177762$

127: 4552: dens 2.120000e-03 $80.26248032830 .00015405029587 .38993841191546 \mathrm{e}-050.0001205067537$

128: 4876: dens 2.120000e-02 $10.073692381690 .00022315603176 .0369782538137 \mathrm{e}-05 \quad 5.06639325936667$

129: 5752: dens 2.120000e-02 $2-2.86484164838854 \mathrm{e}-05 \quad 2.30648227889695 \mathrm{e}-05-2.60163425910552 \mathrm{e}-052$

130: 5197: dens 2.120000e-02 $30.27991440312 .86784508665307 \mathrm{e}-05 \quad 1.67077482722222 \mathrm{e}-05 \quad 1.919339929$

131: 4196: dens 2.120000e-02 4 -2.878384502 0.0038255982940 .00025056185020 .0005456446682

132: 4931: dens 2.120000e-02 $80.26628593850 .0001361423328-1.90757817264078 \mathrm{e}-06 \quad 6.4225089251695$ ?

133: 2228: c_2 2.120000e-03 0.24585440930 .0356523017

134: 3091: dens_pbp 2.120000e-03 0.0046903579290 .0006224185459

135: 2577: chi_dis 2.120000e-03 4.255156350 .591965577

136: 2661: chi_con 2.120000e-03 1.932273226 0.2535277406

137: 2702: chi_tot 2.120000e-03 6.187429576 0.8335570118

138: 2235: c_2 2.120000e-02 0.18659233380 .0263063763

139: 2921: dens_pbp 2.120000e-02 0.033161571760 .00433682243

140: 2522: chi_dis 2.120000e-02 210.245648 27.5173999

141: 2674: chi_con 2.120000e-02 1.295273026 0.1693578934

142: 2583: chi_tot 2.120000e-02 211.54092127 .68655046

143: 2930: c11 2.120000e-03 2.120000e-03 -0.1104551403 0.03423357881

144: 2996: c11 2.120000e-03 2.120000e-02 -0.074847002550 .02385049761$

145: 2995: c11 2.120000e-02 2.120000e-02 -0.067550650170 .02093595165$

146: 1655: c2q 0.46490055690 .08658757331

147: $1625:$ C2B 0.046461334110 .01365810904

148: 1607 : c2Q 0.19018018480 .02491485075

149: 1625: c2I 0.60216395890 .07888918166

150: 0 :

151: 822:p4b3.60 100800

152: $706:$ dens 30500

153: 5205: dens $1.920000 \mathrm{e}-0310.0071944875136 .30219446525084 \mathrm{e}-05-1.116605110551 \mathrm{e}-05 \quad 4.69998830$

154: 5657: dens 1.920000e-03 2 2.78114599285021e-06 $1.86521475939969 \mathrm{e}-05-7.75900870306432 \mathrm{e}-061.4$

155: 5186: dens 1.920000e-03 $30.28052815212 .12130980613477 \mathrm{e}-05-1.43301631595232 \mathrm{e}-051.21841204 \mathrm{~s}$

156: 3995: dens 1.920000 e-03 4 -3.652543197 0.02028380210 .0026774789770 .002279721301

157: 5206: dens 1.920000e-03 $80.26158191987 .72251228284371 \mathrm{e}-05 \quad 6.06588804199118 \mathrm{e}-054.748960624$

158: 4966: dens 1.920000e-02 $10.061732855710 .0001010639091-1.59351744959258 \mathrm{e}-054.184999503410$

159: 5695: dens $1.920000 \mathrm{e}-0224.19838960530189 \mathrm{e}-061.84871352162539 \mathrm{e}-05-2.28439643113081 \mathrm{e}-061.2$

160: 5216: dens 1.920000e-02 $30.28009802412 .07192661297882 \mathrm{e}-05-1.50742553178029 \mathrm{e}-051.29129505 \mathrm{e}$

161: 4126: dens 1.920000e-02 $4-2.852852770 .002386047801-0.0004175340170 .0003968213047$

162: 5151: dens $1.920000 e-0280.2643016298 .76523782865728 \mathrm{e}-05-7.71851758518123 e-06 \quad 3.217972232$ 
163: 2280: c_2 1.920000e-03 0.33853445410 .02653132047

164: 3098: dens_pbp 1.920000e-03 0.0039805566160 .0003083892279

165: 2685: chi_dis $1.920000 \mathrm{e}-032.4715155680 .1953608739$

166: 2631: chi_con 1.920000e-03 2.02087431 0.1559688578

167: 2702: chi_tot $1.920000 \mathrm{e}-034.4923898780 .3501015228$

168: 2266: c_2 1.920000e-02 0.28431215040 .02230623561

169: 2991: dens_pbp 1.920000e-02 0.03415547344 0.002629880564

170: 2641: chi_dis 1.920000e-02 181.3869266 13.97575184

171: 2660: chi_con 1.920000e-02 1.5784226390.1215141603

172: 2648: chi_tot 1.920000e-02 182.9653493 14.09713306

173: 2997: c11 1.920000e-03 1.920000e-03 -0.063360151190 .01109947304$

174: 3072: c11 1.920000e-03 1.920000e-02 -0.045085279140 .009173389637$

175: 3019: c11 1.920000e-02 1.920000e-02 -0.04481774990 .009141577343$

176: 1648 : c2q 0.68325483420 .05602993384

177: $1704:$ c2B 0.089761149920 .008125536636

178: 1612 : c2Q 0.23875444470 .01842156816

179: 1613: c2I 0.74042905950 .05712926499

180: 0 :

181: $825: p 4 b 3.63100800$

182: $706:$ dens 21500

183: 5276: dens 1.700000e-03 $10.0053385591184 .94804488495711 \mathrm{e}-05 \quad 5.80343342533845 \mathrm{e}-05 \quad 5.2320281$

184: 5686: dens 1.700000e-03 $2-2.61686298802039 \mathrm{e}-052.1519719653112 \mathrm{e}-05-3.77285663431942 \mathrm{e}-061.6$

185: 5228: dens 1.700000e-03 $30.2805570302 \quad 2.71057574418698 \mathrm{e}-05-4.73080256390891 \mathrm{e}-07 \quad 1.71705075$

186: 4051: dens 1.700000e-03 $4-3.1190295880 .0220395788-0.0013537641290 .001659849128$

187: 4800: dens 1.700000e-03 8 $0.26094603940 .00010270412024 .57224226943048 \mathrm{e}-054.6519797181142 \mathrm{e}$

188: 4919: dens $1.700000 \mathrm{e}-0210.050263017970 .00011658258875 .86557812332947 \mathrm{e}-05 \quad 5.0918925002156$

189: 5655: dens $1.700000 \mathrm{e}-022-3.05184787920516 \mathrm{e}-05 \quad 2.24685863431509 \mathrm{e}-05 \quad 7.9920015840959 \mathrm{e}-07 \quad 1.6$

190: 5234: dens $1.700000 e-0230.28025320882 .70898691882384 \mathrm{e}-05 \quad 2.54979599736392 \mathrm{e}-07 \quad 1.657788508 \mathrm{~s}$

191: 4191: dens 1.700000e-02 4 -2.782578261 $0.002598461414 \quad 0.00049471072110 .0004265925746$

192: 4849: dens $1.700000 \mathrm{e}-0280.26255602530 .00010535309869 .38626931813888 \mathrm{e}-053.8999421677618 \mathrm{e}$

193: 2315: c_2 $1.700000 \mathrm{e}-03 \quad 0.29515806870 .03556595968$

194: 3096: dens_pbp 1.700000e-03 0.0025140888870 .0002995253943

195: 2655: chi_dis $1.700000 \mathrm{e}-031.1570208860 .1392144232$

196: 2637: chi_con 1.700000 e-03 1.4688453290 .174777619

197: 2695: chi_tot $1.700000 \mathrm{e}-032.6258662150 .3135851476$

198: 2293: c_2 1.700000e-02 0.26435965310 .03191149694

199: 2979: dens_pbp 1.700000e-02 0.023670374740 .002812161198

200: 2558: chi_dis $1.700000 \mathrm{e}-02102.35116612 .16679813$

201: 2653: chi_con 1.700000e-02 1.310400227 0.1556577165

202: 2623: chi_tot $1.700000 \mathrm{e}-02103.661566212 .32234523$

203: 2997: c11 1.700000e-03 1.700000e-03 -0.041183585560 .01208313845$

204: 3002: c11 $1.700000 e-031.700000 e-02-0.039561736930 .01149312491$

205: 2988: c11 1.700000e-02 1.700000e-02 -0.040603890220 .01123028779$

206: 1656: c2q 0.58956613250 .07377654463

207: 1586: c2B 0.081596525670 .0110960071

208: 1607 : c2Q 0.20689765610 .02461434254

209: 1610: c2I 0.63149972290 .07512952292

210: 0 :

211: 831 :p4b3.69 100800

212: 705:dens 34000 
213: 5273: dens 1.500000e-03 $10.0040393322429 .67030788137432 \mathrm{e}-06 \quad 1.43429328015565 \mathrm{e}-053.66233098$ 214: 5682: dens 1.500000e-03 $24.62483150031338 \mathrm{e}-071.71991959018095 \mathrm{e}-05-6.16920195558381 \mathrm{e}-061$. 215: 5157: dens 1.500000e-03 $30.28047459791 .80550500173124 \mathrm{e}-05-4.0514879406301 \mathrm{e}-06 \quad 1.344206329$ 216: 4156: dens $1.500000 \mathrm{e}-034-2.6897348940 .005727544678 \quad 0.0001281384347 \quad 0.000323817282$

217: 5183: dens 1.500000e-03 $80.26044149165 .33586702902174 \mathrm{e}-05-2.16871376803425 \mathrm{e}-052.15985399$ 218: 5258: dens 1.500000e-02 1 0.03963284572 3.54324746674619e-05 $1.46046170697678 \mathrm{e}-053.59423998$ 219: 5708: dens 1.500000e-02 $2-2.0339548412913 \mathrm{e}-06 \quad 1.69514157161792 \mathrm{e}-05-8.39842359687371 \mathrm{e}-061$. 220: 5141: dens 1.500000e-02 $30.28026911631 .81230893480026 \mathrm{e}-05-2.5914711546731 \mathrm{e}-061.330202409$ 221: 4552: dens 1.500000e-02 $4-2.5799382620 .001625499833-4.20528607327315 e-06 \quad 0.0001639138254$ 222: 5212: dens $1.500000 e-0280.26121644925 .08614644506487 e-05-1.93063442506033 e-052.02486075$ 223: 2213: c_2 1.500000e-03 0.36302611660 .0251026811

224: 3086: dens_pbp 1.500000e-03 0.0023166758450 .0001570926188

225: 2767: chi_dis 1.500000 e-03 0.80515393720 .05470286903

226: 2649: chi_con 1.500000e-03 1.542642071 0.1045922253

227: 2701: chi_tot $1.500000 \mathrm{e}-032.3477960090 .1592656157$

228: 2284: c_2 1.500000e-02 0.34531532450 .02387418457

229: 2967: dens_pbp 1.500000e-02 0.022730602690 .001540527605

230: 2628: chi_dis $1.500000 \mathrm{e}-0277.493036675 .253327491$

231: 2672: chi_con 1.500000e-02 1.479670474 0.1002777565

232: 2668: chi_tot $1.500000 \mathrm{e}-0278.972707145 .353579588$

233: 3062: c11 1.500000e-03 1.500000e-03 -0.049734149040 .009937239852$

234: 3061: c11 1.500000e-03 $1.500000 \mathrm{e}-02-0.047879024140 .009666845075$

235: 3031: c11 1.500000e-02 1.500000e-02 -0.04757997350 .009489282599$

236: $1587:$ c2q 0.71677162460 .0522198016

237: 1534: C2B 0.102875040 .008389111359

238: 1506 : c2Q 0.256421470 .01738829155

239: 1558: c2I 0.77578638220 .0526084561

240: 0 :

241: 829:p4b3.76 100800

242: $704:$ dens 51000

243: 5171: dens 1.390000e-03 $10.0033863929584 .3858824343036 e-06-4.110188253707 e-052.617260687$ 244: 5608: dens 1.390000e-03 2 -7.9577046647247e-06 $1.26253197291404 \mathrm{e}-051.6506195673801 \mathrm{e}-057.01 \mathrm{~s}$ 245: 5237: dens 1.390000e-03 $30.28042623011 .30188312432444 \mathrm{e}-05-2.69232784459428 \mathrm{e}-06 \quad 9.48582637$ : 246: 4232: dens $1.390000 \mathrm{e}-034-2.4351747090 .002906930695-0.00023378116120 .0001447737712$

247: 5190: dens $1.390000 \mathrm{e}-0380.26025115174 .36250386386014 \mathrm{e}-05 \quad 3.97425740719564 \mathrm{e}-051.551577649$ 248: 5298: dens $1.390000 e-0210.033592567342 .05407659023028 \mathrm{e}-05-3.98023705767932 \mathrm{e}-052.59096602$ 249: 5681: dens 1.390000e-02 2 -7.8499408697891e-06 1.25876139947001e-05 $1.62006415729382 \mathrm{e}-056.9$ 250: 5191: dens 1.390000e-02 $30.28026828751 .30310722785725 \mathrm{e}-05-3.06317576929114 \mathrm{e}-06 \quad 9.399105378$ 251: 4852: dens 1.390000e-02 4 -2.389995442 $0.0010119359712 .02728353497169 \mathrm{e}-05 \quad 6.81477479397309 \mathrm{e}$ 252: 5140: dens $1.390000 \mathrm{e}-0280.2607054114 .46488478806864 \mathrm{e}-05 \quad 3.36100745117661 \mathrm{e}-05 \quad 1.4634144863$ 253: 2286: c_2 1.390000e-03 0.40920205030 .01780478792

254: 3408: dens_pbp 1.390000e-03 $0.0021413955479 .19484176058335 \mathrm{e}-05$

255: 2778: chi_dis 1.390000 e-03 0.62384157790 .02682441309

256: 2746: chi_con $1.390000 \mathrm{e}-031.5398898890 .06611611926$

257: 2761: chi_tot 1.390000 e-03 2.163731467 0.09292996364

258: 2316: c_2 1.390000e-02 0.39674810740 .01727568789

259: 3036: dens_pbp 1.390000e-02 0.021242358760 .0009117941644

260: 2626: chi_dis 1.390000e-02 61.38166903 2.635514168

261: 2730: chi_con 1.390000e-02 1.511320647 0.06486759368

262: 2675: chi_tot $1.390000 \mathrm{e}-0262.892989682 .700366658$ 
263: 3015: c11 1.390000e-03 1.390000e-03 -0.035494239380 .00561421398$

264: 3080: c11 1.390000e-03 1.390000e-02 -0.034988517720 .005557361588$

265: 3063: c11 1.390000e-02 $1.390000 \mathrm{e}-02-0.034980393410 .005536594208$

266: $1590:$ c2q 0.82031090830 .0369692933

267: 1645: c2B 0.12329788150 .005950814831

268: 1606: C2Q 0.28319281730 .01217218288

269: 1509: c2I 0.853898340 .03670155376

270: 0 :

271: $877: \mathrm{p} 4 \mathrm{c} 3.525100800$

272: 702 : dens 22000

273: 4592: dens 2.400000e-03 $10.031350404060 .00034816970074 .63775830156134 \mathrm{e}-050.0001036895977$

274: 5704: dens 2.400000e-03 2 2.49609431669062e-05 4.64518451408666e-05 -8.35639434820971e-05 4.4

275: 5193: dens 2.400000e-03 $30.28033110962 .76003416724036 \mathrm{e}-05-3.72711753319321 \mathrm{e}-052.04933567$

276: 3932: dens 2.400000e-03 4 -4.358004089 $0.04844146145 \quad 0.017885146320 .02211814239$

277: 4308: dens 2.400000e-03 $80.26947583910 .0003897481454-0.00067333902230 .0003393556368$

278: 4759: dens 2.400000e-02 $10.100582406 \quad 0.000220465296 \quad 9.83772478092252 \mathrm{e}-06 \quad 7.02817592535059 \mathrm{e}-($

279: 5710: dens 2.400000e-02 $2-2.49667430409892 \mathrm{e}-06 \quad 2.65831233551355 \mathrm{e}-05-2.3138601889649 \mathrm{e}-052.2$

280: 5280: dens 2.400000e-02 $30.27949972792 .39762951918938 \mathrm{e}-05-1.78744493393845 \mathrm{e}-051.36570475$

281: 4202: dens 2.400000e-02 4 -2.756975165 $0.004070399887 \quad 0.0002271477749 \quad 0.0006262442214$

282: 4909: dens 2.400000e-02 $80.2729902597 \quad 0.0001601398464-1.56601273010854 \mathrm{e}-05 \quad 4.7723160823724 \mathrm{~s}$

283: 2278: c_2 2.400000e-03 0.11673335140 .04800763546

284: 2989: dens_pbp 2.400000e-03 0.014962692850 .001732750583

285: 2628: chi_dis 2.400000e-03 40.473454584 .752327477

286: 2679: chi_con 2.400000e-03 2.079956497 0.2408707207

287: 2652: chi_tot 2.400000e-03 42.553411084 .985942991

288: 2335: c_2 2.400000e-02 0.087692319920 .01623261631

289: 2989: dens_pbp 2.400000e-02 0.048005239230 .005534938939

290: 2632: chi_dis 2.400000e-02 415.3788642 47.91844462

291: 2669: chi_con 2.400000e-02 1.315829056 0.1516985263

292: 2663: chi_tot 2.400000e-02 416.694693248 .06997822

293: 2959: c11 2.400000e-03 2.400000e-03 -0.36946927540 .09740801812$

294: 2933: c11 2.400000e-03 2.400000e-02 -0.13339054050 .03782114056$

295: 3007: c11 2.400000e-02 2.400000e-02 -0.083565176730 .02451694816$

296: 1585: c2q 0.13535423660 .1817926403

297: 1565: c2B -0.035010148180 .027130755$

298: 1612: c2Q 0.17152090760 .02058650899

299: 1601: c2I 0.60293597830 .07127133812

300: 0 :

301: 873:p4c3.530 100800

302: $706:$ dens 21500

303: 4832: dens 2.400000e-03 $10.02751827440 .00068375433157 .96537002791784 \mathrm{e}-05 \quad 8.8145828468314 \mathrm{e}$

304: 5694: dens 2.400000e-03 $2-4.71237577624245 \mathrm{e}-05 \quad 3.68409644345057 \mathrm{e}-052.18258482312259 \mathrm{e}-053.3$

305: 5221: dens 2.400000e-03 $30.28042324734 .07185542249005 e-05-3.04003784770769 e-052.45690492$

306: 4004: dens 2.400000e-03 4 -4.578574526 $0.09610297903-0.015137342680 .01748810928$

307: 4132: dens 2.400000e-03 $80.2682763614 \quad 0.00033209273 \quad 0.0002756602807 \quad 0.0002473854623$

308: 4873: dens 2.400000e-02 $10.098039472610 .00032276298926 .57573339263456 \mathrm{e}-05 \quad 5.2916180723142$

309: 5706: dens 2.400000e-02 2 1.35486406047956e-05 2.55168957649307e-05 -1.90331479515499e-06 1.5

310: 5107: dens 2.400000e-02 $30.279622883 .43280592663883 e-051.37167917225399 \mathrm{e}-051.83734869891$

311: 4117: dens 2.400000e-02 $4-2.7780897150 .00577529967 \quad 0.0010165554810 .0008665093666$

312: 4940: dens $2.400000 \mathrm{e}-0280.27229603930 .0001805371871-1.38564017986084 \mathrm{e}-05 \quad 5.8839892742358$ 
313: 2304: c_2 2.400000e-03 0.21668538090 .03979947421

314: 2934: dens_pbp 2.400000e-03 0.012959187360 .00157218092

315: 2619: chi_dis 2.400000e-03 31.15873563 4.013574575

316: 2661: chi_con 2.400000e-03 2.156189166 0.2600305088

317: 2644: chi_tot 2.400000e-03 33.31492479 4.236606436

318: 2269: c_2 2.400000e-02 0.11222943590 .01530117076

319: 2994: dens_pbp 2.400000e-02 0.046169751640 .005486247464

320: 2581: chi_dis 2.400000e-02 389.4559749 46.3308403

321: 2663: chi_con 2.400000e-02 1.308286435 0.1554253546

322: 2658: chi_tot 2.400000e-02 390.7642614 46.48587664

323: 2970: c11 2.400000e-03 2.400000e-03 $-0.1779990566 \quad 0.06295347038$

324: 2965: c11 2.400000e-03 2.400000e-02 -0.039638286720 .0246757449$

325: 2996: c11 2.400000e-02 2.400000e-02 -0.055924944520 .01434196054$

326: 1564 : c2q 0.50064003070 .1341616542

327: 1612: c2B 0.032036063080 .01802311154

328: 1582 : c2Q 0.17681030450 .02143174003

329: 1604 : c2I 0.61136981840 .07372158037

$330: 0:$

331: $878: \mathrm{p} 4 \mathrm{c} 3.535100800$

332: $707:$ dens 22500

333: 4976: dens 2.400000e-03 $10.023963049560 .0003132973573-4.26800457507704 \mathrm{e}-06 \quad 9.655649694809$ 334: 5698: dens 2.400000e-03 2 1.74853063846225e-05 5.00895763962031e-05 -9.83184832192891e-05 4.

335: 5147: dens 2.400000e-03 $30.2803979833 \quad 3.01985792039115 e-052.2528693525709 e-062.9149818384$

336: 3962: dens 2.400000e-03 4 -4.767950493 0.053639662850 .013361329940 .01436491374

337: 4236: dens 2.400000e-03 80.26691244280 .00028126531810 .00012580764080 .0001927212797

338: 4986: dens 2.400000e-02 $10.095600316560 .0001509742365-6.66713777876346 e-05 \quad 6.4926831864656$ 339: 5720: dens 2.400000e-02 2 1.90777606444068e-05 2.55561294855002e-05 -4.88865374755889e-05 2.3

340: 5237: dens 2.400000e-02 $30.27961466982 .40338864083712 \mathrm{e}-05-3.18668106561399 \mathrm{e}-06 \quad 1.80007391$

341: 4204: dens 2.400000e-02 4 -2.793428562 0.0037945734290 .00062050794850 .0006557514562

342: 4848: dens 2.400000e-02 $80.27125191360 .000141710048 \quad 8.78476707868186 \mathrm{e}-06 \quad 4.69953376707397 \mathrm{e}$

343: 2345: c_2 2.400000e-03 0.072348540570 .09951959121

344: 2965: dens_pbp 2.400000e-03 0.011582140620 .001305650757

345: 2630: chi_dis 2.400000e-03 23.98108567 2.757627216

346: 2666: chi_con 2.400000e-03 2.304509405 0.2593383269

347: 2641: chi_tot 2.400000e-03 26.28559507 3.006617406

348: 2274: c_2 2.400000e-02 0.10449024170 .01693481514

349: 2992: dens_pbp 2.400000e-02 0.046206819670 .005174678532

350: 2582: chi_dis 2.400000e-02 379.9909703 42.5674821

351: 2665: chi_con 2.400000e-02 1.350157138 0.1511995584

352: 2635: chi_tot 2.400000e-02 381.341127542 .71857431

353: 2906: c11 2.400000e-03 2.400000e-03 -0.47826143340 .2039656665$

354: 2945: c11 2.400000e-03 2.400000e-02 $-0.1917447903 \quad 0.06116627521$

355: 2931: c11 2.400000e-02 2.400000e-02 -0.11022308580 .02616923082$

356: 1663 : c2q -0.12775855910 .3933795977$

357: 1694: c2B -0.068062632340 .05729651739$

358: 1635: c2Q $0.1793889557 \quad 0.02156438499$

359: 1599: c2I 0.62295851450 .07133532347

360: 0 :

361: $874: \mathrm{p} 4 \mathrm{c} 3.540100800$

362: 701 :dens 21000 
363: 4972: dens 2.400000e-03 $10.020749580880 .0002918290345-2.24449061375075 e-067.7228194213868$ 364: 5653: dens 2.400000e-03 2 8.04278276915344e-06 3.76966745047591e-05 $-5.21121826971037 \mathrm{e}-064$. 365: 5261: dens 2.400000e-03 $30.28043473794 .16484452544339 \mathrm{e}-05-4.05637682587157 \mathrm{e}-052.62486873$ 366: 4010: dens 2.400000e-03 4 -4.848869393 0.060337953020 .0030878649670 .01113774332

367: 4546: dens 2.400000e-03 $80.26513209870 .00020004228882 .03390479766052 \mathrm{e}-050.0002337795152$

368: 4848: dens $2.400000 e-0210.092886742370 .0001495608261 .36083910961203 e-05 \quad 5.202392420551736$ 369: 5703: dens 2.400000e-02 2 1.16959858190557e-05 2.58834170221743e-05 -1.05373183676445e-05 2. 370: 5253: dens 2.400000e-02 $30.27965370343 .78635356119426 e-05-1.48109389786723 e-051.64373273$ 371: 4118: dens 2.400000e-02 $4-2.8083789790 .00364983420 .00074342191160 .0008496797807$

372: 4854: dens 2.400000e-02 $80.27014874490 .00010278922132 .76072421563911 \mathrm{e}-05 \quad 4.68956530430026 \mathrm{e}$ 373: 2279: c_2 2.400000e-03 0.18410067850 .03339426044

374: 3051: dens_pbp 2.400000e-03 0.0096337339790 .001187891256

375: 2620: chi_dis 2.400000e-03 17.274836922 .170053764

376: 2601: chi_con 2.400000e-03 2.25126079 0.2772060223

377: 2648: chi_tot 2.400000e-03 19.52609771 2.437721358

378: 2244: c_2 2.400000e-02 0.12313760280 .0176982989

379: 2945: dens_pbp 2.400000e-02 0.043125987530 .00528393399

380: 2611: chi_dis 2.400000e-02 344.586841342 .23064801

381: 2617: chi_con 2.400000e-02 1.3038902 40.1597520689

382: 2641: chi_tot 2.400000e-02 345.8907316 42.39031069

383: 2951: c11 2.400000e-03 2.400000e-03 -0.2574508141 0.05900639477

384: 2954: c11 2.400000e-03 2.400000e-02 -0.10871812870 .02884207098$

385: 2991: c11 2.400000e-02 2.400000e-02 -0.091570479750 .02100217923$

386: $1606:$ c2q 0.28169571980 .1051968419

387: 1707 : C2B 0.0018279875870 .01609883061

388: 1612: c2Q 0.18525119470 .02289760067

389: 1591: c2I 0.62565217110 .07711295324

390: 0 :

391: $879: \mathrm{p} 4 \mathrm{c} 3.545100800$

392: 706 :dens 17000

393: 4974: dens 2.400000e-03 $10.018417791740 .0004284635864-5.88280661206351 e-056.930487452825$ 394: 5646: dens 2.400000e-03 2 -5.92723544924705e-06 $5.84648892490251 \mathrm{e}-052.42121902611869 \mathrm{e}-063$. 395: 5235: dens 2.400000e-03 $30.28050122963 .29346952926817 \mathrm{e}-05-1.05565695001888 \mathrm{e}-053.08554454 \mathrm{~s}$ 396: 4123: dens 2.400000e-03 $4-4.8364595720 .08995566155-0.0078155960680 .008649011068$ 397: 4316: dens 2.400000e-03 $80.26495992620 .0002374169675-0.00013498171430 .0001799675375$

398: 4945: dens 2.400000e-02 $10.090654791570 .0002170152234-1.86003078112209 \mathrm{e}-05 \quad 5.838502390388$ 399: 5745: dens 2.400000e-02 $2-1.59360090591925 \mathrm{e}-05 \quad 4.23472097606841 \mathrm{e}-05-5.24668431329868 \mathrm{e}-072$ 400: 5201: dens 2.400000e-02 $30.27972846142 .55761708275826 e-05 \quad 4.70128053591645 e-062.8606923765$ 401: 4232: dens 2.400000e-02 $4-2.8154804480 .004327835192-0.00031336440990 .0008249583115$ 402: 4817: dens 2.400000e-02 $80.26957036010 .00015125611436 .1440334087196 \mathrm{e}-054.08679382857486 \mathrm{e}$ 403: 2283: c_2 2.400000e-03 0.22092904440 .03933762418 404: 3015: dens_pbp 2.400000e-03 0.0073129467210 .001211891042

405: 2570: chi_dis 2.400000e-03 11.68905333 1.99141369

406: 2673: chi_con 2.400000e-03 1.920358948 0.3171363907

407: 2651: chi_tot 2.400000e-03 13.60941228 2.291996864

408: 2247: c_2 2.400000e-02 0.12233120220 .02142601571

409: 3001: dens_pbp 2.400000e-02 0.035995284890 .005908229171

410: 2621: chi_dis 2.400000e-02 280.7090647 46.08953611

411: 2659: chi_con 2.400000e-02 1.117911354 0.1834816642

412: 2669: chi_tot 2.400000e-02 281.8269761 46.27289959 
413: 2891: c11 2.400000e-03 2.400000e-03 -0.0863520760 .03151606664$

414: 2948: c11 2.400000e-03 2.400000e-02 -0.054883541140 .0155589144$

415: 3009: c11 2.400000e-02 2.400000e-02 -0.061556317890 .01665034869$

416: $1490:$ c2q 0.48878423270 .10096092

417: 1638: c2B 0.040896681420 .01152640943

418: 1608 : C2Q 0.16161823530 .02661638909

419: 1602: c2I 0.52821016490 .08694713336

420: 0 :

421: $875: \mathrm{p} 4 \mathrm{c} 3.550100800$

422: $711:$ dens 17500

423: 4555: dens 2.400000e-03 $10.018078526610 .00030316876062 .00215654512069 \mathrm{e}-050.0001041000923$

424: 5669: dens 2.400000e-03 2 9.99208546760271e-06 3.45766642213979e-05 3.54187431073907e-05 2.9"

425: 5153: dens 2.400000e-03 $30.2804364333 .23339653075924 \mathrm{e}-051.24295922416798 \mathrm{e}-053.1367980928$

426: 4028: dens 2.400000e-03 4 -4.834541216 0.067613175350 .00012802381080 .01197210897

427: 4254: dens 2.400000e-03 $80.26543989630 .0002576541888-0.00016140473790 .000198158223$

428: 4934: dens 2.400000e-02 $10.08966619546 \quad 0.00015339087395 .51467532295553 e-05 \quad 5.7046373124396$

429: 5621: dens 2.400000e-02 2 2.12072648905901e-05 2.7193531079493e-05 -8.01126303670695e-06 2.3

430: 5260: dens $2.400000 e-0230.27966812112 .69897487352562 e-05-5.97632489096986 e-062.48610820$

431: 4114: dens 2.400000e-02 4 -2.792812713 $0.003677403137-0.0014221024740 .001097075933$

432: 4945: dens 2.400000e-02 $80.26996415730 .0001216933094-8.82292947384273 e-05 \quad 4.94834322578188$

433: 2298: c_2 2.400000e-03 0.21255706570 .03613776888

434: 3039: dens_pbp 2.400000e-03 0.0073605429770 .001164782484

435: 2628: chi_dis 2.400000e-03 11.500969171 .848785543

436: 2687: chi_con 2.400000e-03 1.968348924 0.3109651798

437: 2640: chi_tot 2.400000e-03 13.46931809 2.150163694

438: 2266: c_2 2.400000e-02 0.12452311480 .02093071076

439: 2973: dens_pbp 2.400000e-02 0.036506951010 .005745718113

440: 2610: chi_dis 2.400000e-02 281.5820955 44.32501434

441: 2672: chi_con 2.400000e-02 1.137073747 0.1789563811

442: 2650: chi_tot 2.400000e-02 282.7191693 44.50391279

443: 3027: c11 2.400000e-03 2.400000e-03 -0.087876179030 .02857156799$

444: 3002: c11 2.400000e-03 2.400000e-02 -0.048788521840 .01831309641$

445: 2884: c11 2.400000e-02 2.400000e-02 -0.050321997090 .016420543$

446: 1656: c2q 0.45946136470 .09068907365

447: 1634: c2B 0.040464891480 .01147245304

448: 1543: c2Q 0.1568721470 .02480060044

449: 1591: c2I 0.51299031030 .08105774813 